\title{
CT Findings in Patients Affected by H1N1 Influenza Virus
}

\author{
Marirosa Cristallo Lacalamita, Emanuela Salinaro, Maria Raffaella Abrusci, \\ Marco Moschetta, Giuseppe Angelelli \\ Interdisciplinay Department of Medicine, Section of Radiology, Medical School, University of Bari, Bari, Italy \\ Email: marirosacristallo@libero.it
}

Received January 12, 2012; revised February 14, 2012; accepted February 23, 2012

\begin{abstract}
Objectives: To evaluate the primary lesions, the complications and the evolution of disease in patients affected by H1N1 viral infection. Materials and Methods: 24 Patients affected by H1N1 infection, diagnosed by polymerase chain reaction (PCR) on throat swabs, underwent CT examination. Seven patients were hospitalized in intensive care unit (ICU). In five patients the evolution of disease was monitored. The following features were evaluated: primary lesions significant for viral infection, their possible complications and the evolution of disease in controlled patients. Results: Primary lesions variously associated with each other were found in 22 out of 24 patients: ground glass opacities (19/24, $79.2 \%)$, interstitial thickening (13/24, 54.2\%), centrilobular nodules $(3 / 24,12.5 \%)$ and consolidation $(8 / 24,33.3 \%)$. The following complications were observed: 3 consolidations with air bronchogram, 9 pleural effusions, 7 ARDS and 1 barotrauma. In the 5 patients who underwent follow-up (including 3 admitted to ICU), complete resolution was demonstrated in 4 cases and focal fibrotic evolution in one case. 3 ICU patients affected by ARDS died. Conclusions: In case of H1N1 virus infection. CT is an important tool for staging the disease, recognize complications and to study disease evolution.
\end{abstract}

Keywords: Computed Tomography; CT-H1N1; Virus; Infection

\section{Introduction}

H1N1 virus is a RNA virus belonging to the family of Orthomyxoviridae. There are two ways of spread for influenza: the endemic form caused by the $\mathrm{B}$ group and the pandemic one supported by the A group, such as the "Spanish" occurred in 1918-1919, the "Asiatic" in 19571958 and the "Hong-Kong" in 1968-1969. The H1N1 virus is a type A influenza virus with swine origin (SOIV, swine origin influenza virus). It infects wide varieties of warm-blooded animals, including birds, swine, horses, and humans, whereas influenza B and influenza $\mathrm{C}$ viruses almost exclusively infect humans and are also implicated in epidemics. The large, dynamic reservoir of influenza A subtypes in animals, as well as their evolutionary adaptability, help produce new influenza strains that cause new epidemics and pandemics [1].

The first inter-human infection was reported in Mexico in April 2009 [2] and the rapid spread around the world was such that in June 2009 the WHO (World Health Organization) declared the pandemic state, as a scale 6 global emergency [3].

In Italy the spread of the H1N1 virus was observed in association with the spread of the virus that caused seasonal influenza in 2009-2010 and 2010-2011 [4]. Children, young adults and elderly subjects represent the most affected groups, while the population at high risk of morbidity and mortality is represented by children, pregnant women, patients affected by heart disease, chronic respiratory diseases and immune-compromised subjects [5].

The clinical manifestations of the disease are various: asymptomatic forms, mild involvement of the upper airways (dyspnea, fever, coughing) and gastrointestinal system (diarrhea and nausea), severe pneumonia with acute respiratory distress syndrome (ARDS), up to multi-organ failure and exitus.

Measures of hygiene and personal protection play an important role in order to limit the spread of the disease. Influenza vaccination represents an effective and safe way to prevent the disease and its complications in patients at risk. Antiviral treatments have been applied to patients with documented H1N1 infection [4], but in case of severe clinical settings with respiratory failure, it is better to hospitalize the patient in order to get ventilatory therapy [5].

The diagnosis of H1N1 infection is usually suspected basing on clinical data, but it must be confirmed by RTPCR (reverse transcriptase polymerase chain reaction) on throat swab [3].

Diagnostic imaging based on chest radiography and computed tomography (CT) is very useful in the study of disease because it allows to assess the extent of lung pa- 
renchymal damage.

The purpose of this paper is to evaluate the role of CT in the study of the disease, and especially to identify the primary injury, to research any complication and to consider the evolution of disease.

\section{Materials and Methods}

CT examinations of 24 patients studied between November 2009 and March 2011 and affected by H1N1 influenza were retrospectively reviewed. Diagnosis was confirmed by RT-PCR technique performed on viral genetic material extracted from throat swabs.

Two thoracic radiologists (blinded) with more than 5 years of experience reviewed recent literature and performed the image analysis independently. Difference in assessment were resolved by consensus. Each radiologist evaluated TC examinations in a random order.

The examined sample consisted of 12 females (50\%) and 12 males (50\%), aged between 20 and 73 years (mean 46, median 47.5).

All subjects were hospitalized at the time of the study: 11 out of $24(45.8 \%)$ in departments of pulmonary medicine, 5 out of $24(20.8 \%)$ in departments of infectious diseases, one patient (4.2\%) in haematology ward.

7 out of 24 patients (29.2\%), in critical condition, were admitted to the Intensive Care Unit (ICU) and received mechanical assisted ventilation (MAV).

$45.8 \%$ of total (11 out of 24 patients) had variously associated co-morbidities: 4 out of 11 patients (36.3\%) had hypertension, 4 (36.3\%) were allergic, 3 (27.3\%) were affected by heart disease, 2 (18.2\%) were diabetics, $2(18.2 \%)$ had received kidney transplant. One patient (9.1\%) was affected by deep vein thrombosis (DVT), autoimmune thyroiditis, Down's Syndrome, Kaposi's sarcoma, acute myeloid leukemia (AML) and severe chronic obstructive pulmonary disease (COPD). Finally, one patient was obese and affected by neurological deficits.

19 patients underwent only one CT examination, 5 were monitored over time for the persistence of clinical significant signs.

CT examinations were performed by using a CT device equipped with a 16-slice acquisition system (Aquilion TSX-101A-16, Toshiba Medical Systems, Tokyo, Japan) and a CT devise equipped with a 320-slice acquisition system (Aquilion One, Toshiba Medical Systems, Ottawara, Japan).

The scanning protocol consisted in $1 \mathrm{~mm}$ slice thickness, $\mathrm{kV} / \mathrm{mAs} 120 / 250$, pitch 1.75 , increment 0.8 , tube rotation time $0.5 \mathrm{~s}$ for the 16-row CT system, while a protocol of $0.5 \mathrm{~mm}$ slice thickness, $\mathrm{kV} / \mathrm{mAs} 120 / 250$, pitch 1.25 , increment 0.5 , tube rotation time $0.5 \mathrm{~s}$ was used for the 320-row CT system.

In collaborating patients, CT scans were performed in deep inspiration phase.
In 23 cases unenhanced CT scans were acquired; in a single case, $110 \mathrm{~mL}$ of contrast medium (Bracco Iomeron 470 , Milan, Italy) at a flow rate of $3 \mathrm{~mL} / \mathrm{s}$ were intravenously injected because of a clinical suspicion of pulmonary embolism.

The scan delay was calculated by using the Sure Start technique.

All CT examinations were transferred and evaluated on a workstation (HP XW 8600) equipped with a software dedicated to image reconstruction (Vitrea FX 2.1, Vital Images, Minneapolis, Minnesota, US). CT transverse images and multi-planar reconstructions (MPR) were analyzed by two independent blinded radiologists, with 30 and 5 years of experience in the field of thoracic imaging and image reconstruction software; in case of nodular lesions, the Maximum Intensity Projection (MIP) program with a reconstruction thickness of $7 \mathrm{~mm}$ was used in order to obtain a more accurate identification of nodular lesions.

In all cases, CT images were evaluated with a window for the study of mediastinal structures (width $350 \mathrm{HU}$, window $40 \mathrm{HU}$ ) and for the study of lung parenchyma (width $1500 \mathrm{HU}$, window -700 HU). Differences in assessment were resolved by consensus. The duration of post-processing was approximately 15 minutes.

All cases were examined searching for primary lesions significant for viral infection by referring to the glossary of the Fleischner Society [6]: ground glass opacities (inhomogeneous increase in parenchymal density, with preservation of the bronchial and vascular profiles), consolidation (increase in parenchymal density with disappearance of bronchial and vascular structures), interstitial thickenings, nodules (opacities with a maximum diameter of $3 \mathrm{~cm}$ ).

In order to assess any early or late complication in patients who underwent follow-up, the following parameters were considered: bacterial super-infection (consolidation with air bronchogram), bronchiectasis, pleural effusion, lymphadenopathy, ARDS, barotrauma due to mechanical ventilation, fibrosis.

Basing on their distribution, the lung lesions were considered diffuse, when extended to more than one lobe, or lobar.

\section{Results}

Overall, CT examination recognized lung disorders in 22 out of 24 patients (91.7\%).

In particular, the following elementary lesions have been identified: ground glass opacities in $79.2 \%$ of cases $(19 / 24)$, consolidation in $33.3 \%$ of cases $(8 / 24)$, interstitial thickening in $54.2 \%$ of cases (13/24), nodules in $12.5 \%$ of cases $(3 / 24)$.

The ground glass opacities were diffuse in 12 out of 19 cases (63.1\%) and lobar in 7 out of 19 cases (36.8\%). In 
particular, diffuse lesions had sub-pleural distribution in 7 out of 12 cases (58.3\%) and peribronchovascular distribution in 5 out of 12 cases (41.7\%). Lobar lesions were located in the right upper lobe in three cases $(3 / 7,42.8 \%)$, left lower lobe in two $(2 / 7,28.6 \%)$, right lower lobe in one $(1 / 7,14.3 \%)$ and left upper lobe in one patient $(1 / 7$, $14.3 \%)$.

The ground glass opacities occurred as an isolated form in 3 out of 19 cases (15.8\%), associated with parenchymal consolidation in 3 out of 19 cases (15.8\%), associated with interstitial thickening in 9 out of 19 cases (47.4\%) (Figure 1) and associated with both consolidations and interstitial thickening in 4 out of 19 cases (21.05\%).

Parenchymal consolidations were observed in 8 patients, and ground glass opacities were associated in 7 cases (Figure 2). In $75 \%$ of cases (6/8) consolidations were diffuse and in $25 \%$ of cases (2/8) occurred as focal consolidations: one located in the middle lobe and one in the right lower lobe.

A variable degree of interstitial thickening responsible for a reticular appearance was identified in 13 out of 24 patients (54.2\%) and it was always associated with ground glass opacities. The inter-lobular interstitium was involved in all cases $(13 / 13,100 \%)$, associated with intra-lobular interstitial involvement in 9 out of 13 cases (69.2\%). The sub-pleural interstitium was involved in 7 out of 13 cases $(53.8 \%)$.

Nodules with a diameter ranging from $5 \mathrm{~mm}$ and $1 \mathrm{~cm}$ were found in 3 out of 24 cases (12.5\%) (Figure 2).

In the single patient who underwent contrast examination no pulmonary perfusion abnormalities were demonstrated.

Among the complications, the following findings were observed: 3 bacterial super-infections (3/24, 12.5\%), 9 pleural effusions (9/24, 37.5\%), 7 ARDS (7/24, 29.2\%), associated with barotrauma in one case $(1 / 7,14.3 \%)$ (Figure 3). Bacterial complications had a multi-segmental distribution in all cases; they were located at the right upper lobe in two cases and at the left lower lobe in one case.

Pleural effusion was detected in 9 cases (9/24, 37.5\%); it was bilateral in 5 cases $(5 / 9,55.5 \%)$ and unilateral in 4

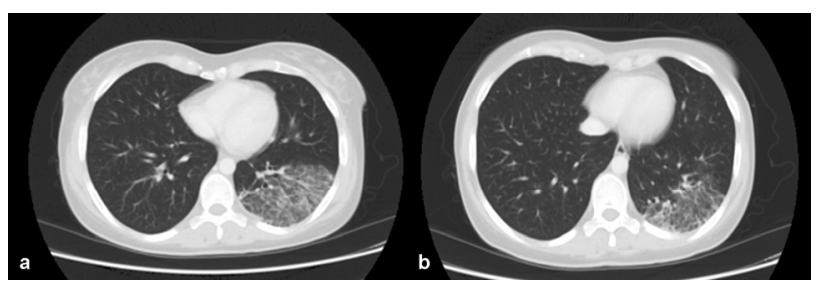

Figure 1. Patient affected by H1N1 infection (a, b). CT transverse scans. Ground glass areas with multi-segmental distribution can be detected in the left lower lobe; intra and inter-lobular interstitial thickening is associated.

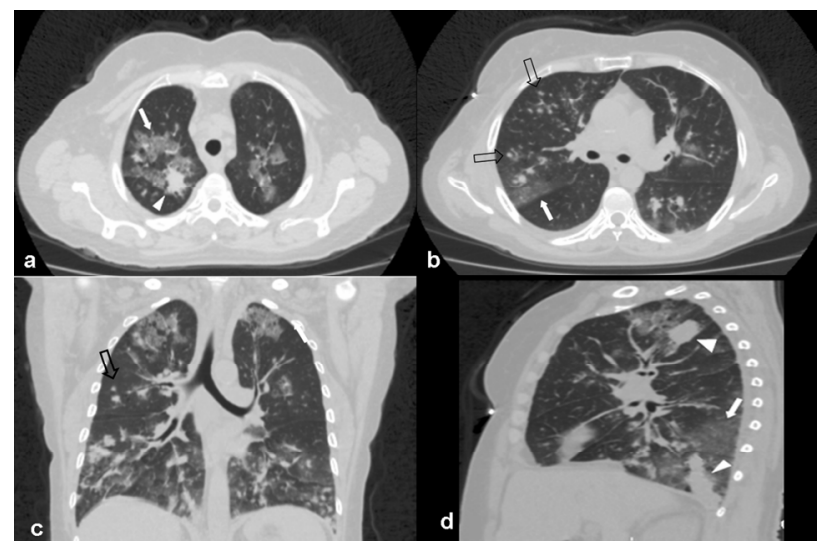

Figure 2. Patient affected by H1N1 infection. Ground glass areas (arrows) (CT transverse scans, (a)-(b); MPR reconstruction on coronal plane, (c); MPR reconstruction on sagittal plane, (d); consolidations (arrowheads) (a), (d) and nodules (empty arrows) (b), (c) can be observed.

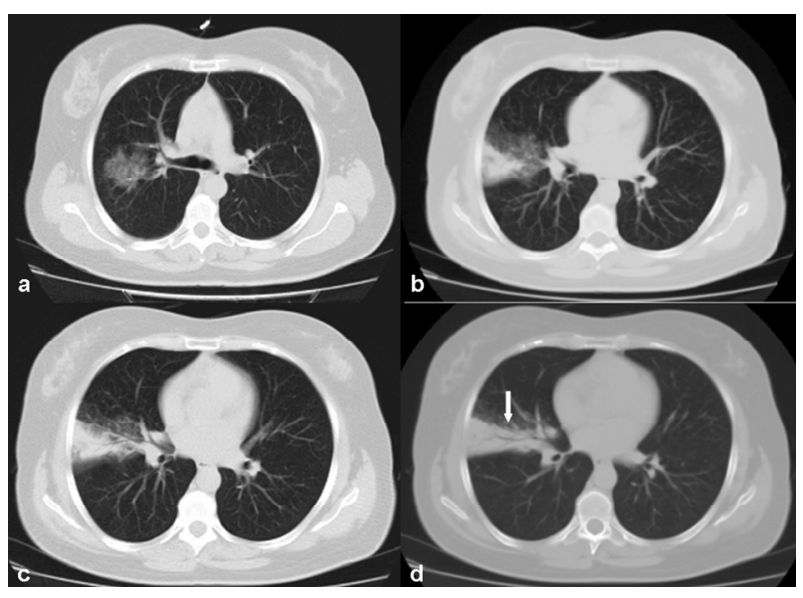

Figure 3. Patient affected by H1N1 infection, complicated by bacterial super-infection. CT transverse scans. Presence of ground glass areas (a) associated with parenchymal consolidations (b), (c) and air bronchogram (arrow) (d).

cases (4/9, 44.4\%), right located in 2 cases (2/4, 50\%) and left located in the remaining 2 cases (2/4, 50\%).

Besides, in the two patients with basal left pleural effusion, it represented the only reported finding.

CT control examinations were performed in 5 patients (5/24, 20.8\%), including 3 affected by ARDS (3/5 60\%). In $4(4 / 5,80 \%)$ cases a complete resolution of the disease was observed.

A gradual evolution of parenchymal damage was found in one case. At the time of admission, multiple areas of ground glass opacities and parenchymal consolidations within a CT setting significant for ARDS were identified. Then, interstitial emphysema with "ring around the artery" sign, pneumo-thorax, pneumo-mediastinum and subcutaneous emphysema, probably induced by mechanical assisted ventilation (MAV), were observed (Figure 4).

These alterations were determined by barotrauma and 


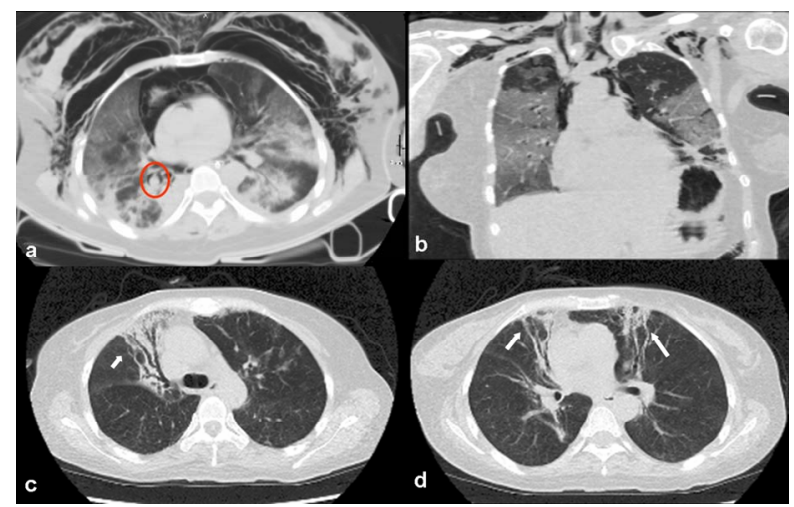

Figure 4. Patient affected by H1N1 infection complicated with ARDS and barotrauma (a) transverse CT scan; (b) coronal reconstruction). Same patient: evolution of the disease (c)-(d) transverse CT scan). Presence of ground glass areas mainly located on upper lobes and parenchymal consolidations in lower lobes. Interstitial emphysema (ring sign) (red circle), pneumo-mediastinum, pneumo-neck and subcutaneous emphysema are associated (a), (b). 45 days later, the appearance of fibrotic streaks and traction bronchiectases (arrows) in the upper lobes (c), (d) can be observed, associated with the regression of all other parenchymal lesions.

progressively decreased. 45 days later, a complete resolution of parenchymal consolidations was observed together with the development of fibrosis in the ground glass areas (Figure 4).

With regard to the clinical outcome in the reported series, in 21 patients $(21 / 24,87.5 \%)$ complete recovery occurred and exitus in $3(3 / 24,12.5 \%)$ : an obese patient affected by autoimmune thyroiditis, a patient affected by hypertension and with kidney transplant and the last one affected by Down's syndrome and severe COPD.

\section{Discussions}

Since 2009, the first era of inter-human infection occurred in Mexico, a rapid worldwide spread of H1N1 has been observed and its pandemia has become a global emergency. The diagnosis of this infection is based on clinical signs and laboratory data, especially on the RTPCR of throat swab. In fact, clinical manifestation range from asymptomatic forms up to respiratory failure and multi-organ failure. As outlined in literature, after obtaining a diagnosis, the assessment of the extent of pulmonary parenchymal damage by means of chest radiograph and/or chest CT examination is mandatory. According to some authors [7], chest radiograph is sufficient to make the diagnosis and CT scan may be reserved for selected cases, some others instead [8-10] emphasize the use of $\mathrm{CT}$ as a more accurate tool in defining the type and distribution of lesions, arguing that X-ray investigation can sometimes underestimate the damage.

In this respect, the results reported by various authors regarding the type of primary lesions are essentially the same, although their incidence is variable in different series.

In the reported experience, ground glass opacities represent the most frequent alteration, with a 79.2\% (19/24) incidence value.

This result corresponds to upper limits of the range values reported in literature [7-13], ranging from 12\% [7] and $65 \%$ [10]. The detection of ground glass opacity areas is not specific for influenza pneumonia because this feature could be secondary to parenchymal lung infectious or noninfectious diseases. However, the recognition of this finding in symptomatic patients is important because it is reported that ground glass opacity of peripheral areas may represent an early sign of H1N1 infection [14].

Parenchymal consolidations were observed in 33.3\% of cases (8/24). Their distribution was diffuse in $75 \%$ of cases and focal in $25 \%$. As reported in literature, incidence values regarding consolidations widely range from between 5\% [7] and 85\% [10].

A reticular interstitial thickening was noted in $54 \%$ of cases, with an incidence value higher than those reported so far in literature, that run between 3\% [8] and 37\% [7]. Probably this difference is due to the severity of the considered patients, as the reticular appearance is likely to be considered indicative of a more advanced stage of disease.

Nodules were found in $12.5 \%$ of patients, and that value is placed among the lowest percentage of those reported in literature, ranging from $4.8 \%$ [12] to $40 \%$ [10].

Complications reported in the study were: 3 cases of bacterial super-infection (3/24, 12.5\%), 9 pleural effusion (9/24, 37.5\%), 7 ARDS (7/24, 29.2\%), 1 barotrauma (1/7, $14.3 \%)$.

In the reported research, bacterial complications were observed in $12.5 \%$ of cases and, as reported in literature $[15,16]$, their recognition was based on the detection of consolidations with air bronchogram, associated with pleural effusion in one case. In contrast, there were no other signs suggestive of bacterial infection such as tree in bud opacities, pericardial effusion, hilar adenopathy, cavitation and hydro-pneumo thorax [11].

Pleural effusion was found in $37.5 \%$ of patients, a percentage that ranks among the highest values reported in literature, ranging from $10 \%$ [7] to $44.4 \%$ [11] of cases.

The presence of pleural effusion was reported in many series in literature [11-13]: some authors correlate this sign to the bacterial infectious complications of the underlying disease [11], other [13] instead consider pleural effusion as a H1N1 infection recognizable injury.

$29.1 \%$ of patients (7/24) presented radiological and clinical findings specific for ARDS [17], which required admission to ICU; this represents a complication reported 
in numerous studies with percentages ranging from $7.5 \%$ [7] and 25\% [13].

20.8\% (5/24) of patients, including 3 affected by ARDS, underwent CT follow-up examinations and in 4 of them a complete regression of the disease was observed. In a single case, interstitial emphysema, pneumothorax, pneumo-mediastinum and subcutaneous emphysema related to the development of barotrauma were detected, probably caused by mechanical assisted ventilation; this represents a complication reported by some authors [18], with a frequency ranging from $17 \%$ [13] to $62 \%$ of cases [12].

The following controls in the same patient showed the occurrence of focal fibrosis, considered secondary to H1N1 infection because of its characteristics, being occurred at a time distance of 45 days from hospitalization, in contrast to the expected fibrosis after ARDS, in which the damage occurs at a time distance of about 7 days [17-19].

Recently, the role of some viral infections (Hepatitis C Virus, Epstein Barr Virus, Cytomegalovirus and H1N1) in the pathogenesis of idiopathic pulmonary fibrosis (IPF) has been emphasized [20] and, in particular, it has been shown that in some subjects such viruses can modulate the ELMOD2 gene involved in the fibroblast response to viral infection at epithelial cells and alveolar macrophages and activate the pathogenetic mechanism of IPF [21].

In the considered series, a complete recovery was found in $87.5 \%$ of patients and exitus in $12.5 \%$ and these values are comparable with those reported in literature, providing recovery rates between 50\% [10] and 97.5\% [7] and exitus between 2.5\% [7] and 40\% [11].

It should be remarked that all patients who died had important comorbidities, and probably their exitus could be related to the presence of associated diseases (autoimmune thyroid disease, hypertension, renal transplant, Down's syndrome, severe COPD), rather than to the extent of parenchymal damage, as also shown in previous experiences reported by other authors $[2-4,7,10]$.

\section{Conclusions}

In patients affected by H1N1 infection, CT examination represents a feasible tool because it is accurate in showing the extent of parenchymal damage and the evolution of the disease.

The main limitation of this technique remains the inability to characterize the H1N1 infection according to the type of primary lesions, but probably further experiences on larger series will improve the so far obtained results.

\section{REFERENCES}

[1] G. Watts, "Pandemic Flu A/H1N1 Influenza Virus: The
Basics,” July 2009.

http://www.bmj.com/content/339/bmj.b3046

[2] R. Perez-Padilla, D. de la Rosa-Zamboni, S. Ponce de Leon, et al., "Pneumonia and Respiratory Failure from Swine-Origin Influenza A (H1N1) in Mexico," The New England Journal of Medicine, Vol. 361, No. 7, 2009, pp. 680-689.

[3] World Health Organization, "Human Infection with Pandemic (H1N1) 2009 Virus: Updated Interim WHO Guidance on Global Surveillance,” July 2009.

http://www.who.int/csr/resources/publications/swineflu/in terim_guidance/en/index.html

[4] Circolare del Ministero della Salute, "Prevenzione e Controllo Dell'Influenza: Raccomandazioni per la Stagione 2010-2011,” July 2010.

http://www.normativasanitaria.it/normsan-pdf/0000/3486 1_1.pdf

[5] Centers for Disease Control and Prevention "People at High Risk of Developing Flu-Related Complications,” November 2009. http://www.cdc.gov/h1n1flu/highrisk.htm.

[6] D. M. Hansell, A. A. Bankier, H. MacMahon, B. Bao, T. C. McLoud, N. L. Müller and J. Remy, "Fleischner Society: Glossary of Terms for Thoracic Imaging,” Radiology, Vol. 246, No. 3, 2008, pp. 697-722. doi:10.1148/radiol.2462070712

[7] E. Busi Rizzi, V. Schininà, F. Ferraro, L. Rovighi, M. Cristoforo, D. Chiappetta, F. Lisena, F. Lauria and C. Bibbolino, "Radiological Findings of Pneumonia in Patients with Swinw-Origin Influenza A Virus (H1N1)," La Radiologia Medica, Vol. 115, No. 4, 2010, pp. 507515. doi:10.1007/s11547-010-0553-9

[8] P. Agarwal, S. Cinti and E. A. Kazerooni, "Chest Radiographic and CT Findings in Novel Swine-Origin Influenza A (H1N1) Virus (S-OIV) Infection,” American Journal of Roentgenology, Vol. 193, No. 6, 2009, pp. 14881493. doi:10.2214/AJR.09.3599

[9] A. M. Ajlan, B. Quiney, S. Nicolaou and N. L. Müller, "Swine-Origin Influenza A (H1N1) Viral Infection: Radiographic and CT Findings,” American Journal of Roentgenology, Vol. 193, No. 6, 2009, pp. 1494-1499. doi:10.2214/AJR.09.3625

[10] B. M. Elicker, B. S. Schwartz, C. Liu, E. C. Chen, S. A. Miller, C. Y. Chiu and W. Richard Webb, “Thoracic CT Findings of Novel Influenza A (H1N1) Infection in Immunocompromised Patients,” Emergency Radiology, Vol. 17, No. 4, 2010, pp. 299-307. doi:10.1007/s10140-010-0859-x

[11] M. Coppola, A. Porto, D. De Santo, S. De Fronzo, R. Grassi and A. Rotondo, "Influenza A Virus: Radiological and Clinical Findings of Patients Hospitalised for Pandemic H1N1 Influenza,” La Radiologia Medica, Vol. 116, No. 5, 2011, pp. 706-719. doi:10.1007/s11547-011-0622-0

[12] T. Valente, F. Lassandro, M. Marino, F. Squillante, M. Aliperta and R. Muto, "H1N1 Pneumonia: Our Experience in 50 Patients with a Severe Clinical Course of Novel Swine-Origin Influenza A (H1N1) Virus (S-OIV)," La Radiologia Medica, Vol. 116, No. 7, 2011, pp. 989- 
1152.

[13] G. Mineo, F. Ciccarese, C. Modolon, M. P. Landini, M. Valentino and M. Zompatori, "Post-ARDS Pulmonary Fibrosis in Patients with H1N1 Pneumonia: Role of Follow-Up CT,” La Radiologia Medica, Vol. 116, No. 7, 2011, pp. 989-1152.

[14] D. J. Mollura, D. S. Asnis, R. S. Crupi, et al., "Imaging Findings in a Fatal Case of Pandemic Swine-Origin Influenza A (H1N1),” American Journal of Roentgenology, Vol. 193, No. 6, 2009, pp. 1500-1503. doi:10.2214/AJR.09.3365

[15] J. Vilar, M. L. Domingo, C. Soto and J. Cogollos, "Radiology of Bacterial Pneumonia,” European Journal of Radiology, Vol. 51, No. 2, 2004, pp. 102-113. doi:10.1016/j.ejrad.2004.03.010

[16] S. Sharma, B. Maycher and G. Eschun, "Radiological Imaging in Pneumonia: Recent Innovations," Current Opinion in Pulmonary Medicine, Vol. 13, No. 3, 2007, pp. 159-169. doi:10.1097/MCP.0b013e3280f3bff4

[17] G. R. Bernard, A. Artigas, K. L. Brigham, J. Carlet, K. Falke, L. Hudson, M. Lamy, J. R. Legall, A. Morris and R. Spragg, "The American-European Consensus Conference on ARDS. Definitions, Mechanisms, Relevant Out- comes, and Clinical Trial Coordination,” American Journal of Respiratory and Critical Care Medicine, Vol. 149, No. 3, 1994, pp. 818-824.

[18] H. Henry Guo, R. T. Sweeney, D. Regula and A. N. Leung, "Fatal 2009 Influenza A (H1N1) Infection, Complicated by Acute Respiratory Distress Syndrome and Pulmonary Interstitial Emphysema,” Radio Graphics, Vol. 30, 2010, pp. 327-333. doi:10.1148/rg.302095213

[19] M. Prokop and M. Galanski, “Tomografia Spirale e Multistrato,” Elsevier-Masson, Milano, 2006.

[20] R. Kaarteenaho1 and V. L. Kinnula Hindawi, "Diffuse Alveolar Damage: A Common Phenomenon in Progressive Interstitial Lung Disorders,” Hindawi Publishing Corporation Pulmonary Medicine, Vol. 2011, 2011, Article ID: 531302, pp. 1-10.

[21] V. Pulkkinen, S. Bruce, J. Rintahaka, U. Hodgson, T. Laitinen, H. Alenius, V. L. Kinnula, M. Mylla"rniemi, S. Matikainen and J. Kere, "ELMOD2, a Candidate Gene for Idiopathic Pulmonary Fibrosis, Regulates Antiviral Responses,” The FASEB Journal Research Communication, Vol. 24, No. 4, 2010, pp. 1167-1177. doi:10.1096/fj.09-138545 